\title{
An update on PET-based molecular imaging in neuro-oncology: challenges and implementation for a precision medicine approach in cancer care
}

\author{
Hossein Shooli ${ }^{1}$, Habibollah Dadgar², Yi-Xiáng J Wáng ${ }^{3}$, Manochehr Seyedi Vafaee ${ }^{4,5,6}$, \\ Saman Rassaei Kashuk ${ }^{1}$, Reza Nemati ${ }^{7}$, Esmail Jafari ${ }^{1}$, Iraj Nabipour ${ }^{8}$, Ali Gholamrezanezhad ${ }^{9}$, \\ Majid Assadi ${ }^{1}$, Mykol Larvie $^{10,11}$
}

${ }^{1}$ The Persian Gulf Nuclear Medicine Research Center, Department of Molecular Imaging and Radionuclide Therapy (MIRT), Bushehr Medical University Hospital, Faculty of Medicine, Bushehr University of Medical Sciences, Bushehr, Iran; ${ }^{2}$ Cancer Research Center, RAZAVI Hospital, Imam Reza International University, Mashhad, Iran; ${ }^{3}$ Department of Imaging and Interventional Radiology, Faculty of Medicine, The Chinese University of Hong Kong, Prince of Wales Hospital, Shatin, New Territories, Hong Kong SAR, China; ${ }^{4}$ Department of Nuclear Medicine, Odense University Hospital, Odense, Denmark; ${ }^{5}$ Translational Neuroscience, BRIDGE, University of Southern Denmark, Odense, Denmark; ${ }^{6}$ Neuroscience Research Center, Faculty of Medicine, Tabriz University of Medical Sciences, Tabriz, Iran; ${ }^{7}$ Department of Neurology, Bushehr Medical University Hospital, Faculty of Medicine, Bushehr University of Medical Sciences, Bushehr, Iran; ${ }^{8}$ The Persian Gulf Marine Biotechnology Research Center, The Persian Gulf Biomedical Sciences Research Institute, Bushehr University of Medical Sciences, Bushehr, Iran; ${ }^{9}$ Department of Diagnostic Radiology, Keck School of Medicine, University of Southern California, Los Angeles, CA 90033, USA; ${ }^{10}$ Department of Nuclear Medicine, ${ }^{11}$ Neurological Institute, Cleveland Clinic, Cleveland, OH 44195, USA

Correspondence to: Majid Assadi, MD. The Persian Gulf Nuclear Medicine Research Center, Bushehr University of Medical Sciences, Bushehr, Iran. Email: asadi@bpums.ac.ir; assadipoya@yahoo.com.

\begin{abstract}
PET imaging using novel radiotracers show promises for tumor grading and molecular characterization through visualizing molecular and functional properties of the tumors. Application of PET tracers in brain neoplasm depends on both type of the neoplasm and the research or clinical significance required to be addressed. In clinical neuro-oncology, ${ }^{18} \mathrm{~F}-\mathrm{FDG}$ is used mainly to differentiate tumor recurrence from radiation-induced necrosis, and novel PET agents show attractive imaging properties. Novel PET tracers can offer biologic information not visible via contrast-enhanced MRI or ${ }^{18} \mathrm{~F}$-FDG PET. This review aims to provide an update on the complementary role of PET imaging in neuro-oncology both in research and clinical settings along with presenting interesting cases in this context.
\end{abstract}

Keywords: Positron emission tomography (PET); brain tumors; magnetic resonance imaging (MRI); precision medicine; molecular imaging; ${ }^{68} \mathrm{Ga}$-DOTA-peptide; ${ }^{68} \mathrm{Ga}$-labeled PSMA

Submitted Jul 02, 2019. Accepted for publication Aug 19, 2019.

doi: 10.21037/qims.2019.08.16

View this article at: http://dx.doi.org/10.21037/qims.2019.08.16

\section{Introduction}

Brain neoplasms include primary brain neoplasms which stem from the brain parenchyma, and metastatic lesions originating from other primary malignancies outside of the brain. The former is less frequent than the latter. According to the American Cancer Society report, approximately 24,000 of new cases are diagnosed with primary brain neoplasms every year while about 17,000 death occurs due to primary brain tumors every year in USA $(1,2)$. Brain metastases comprise more than half of intracranial tumors in adults (3), with incidence of $9 \%$ to $17 \%$ in patients with cancer (4). Tumors of the brain and the other parts of the nervous system are the most leading cause of death from cancer among males before 40 years of age and females aged 
less than 20 years (2). The most common sites of origination include lung, breast, and skin (melanoma). Moreover, in about $15 \%$ of metastatic brain lesions, the primary site of the tumor remains undiscovered (3).

The most common types of primary brain tumors include glioma which is the most lethal brain tumor, accounting for about $45 \%$ to $50 \%$ of total primary brain tumors, followed by meningiomas, the second commonest, and comprising nearly $15 \%$ of all the primary brain neoplasms. The other types of primary brain tumors occur with less incidence $(5,6)$. Gliomas are divided into several groups including astrocytoma, oligodendroglioma, choroid plexus tumors, ependymal tumors, and embryonal tumors (1). According to the WHO Classification of Tumors of the Central Nervous System (CNS) 2016 (1), tumors are graded into four grades based on the histopathologic characteristics in which grade 1 represents benign features and grade 4 indicates the most malignant tumors (1). Current practice in tumor grading relies on histologic studies that require tissue biopsy of brain neoplasms. The potential complications during and after the procedure and the invasive nature of tissue biopsy remain the main challenges of tumor grading. Recently, the emergence of novel technologies, such as molecular genomics, novel tracers and imaging techniques, offer the potential to identify the molecular properties and grading of the neoplastic tissues noninvasively. Positron emission tomography (PET) modality with a wide range of radiotracers may have the capacity as a complementary imaging modality to routine clinical practice.

Chemotherapy, radiotherapy, surgical resection, and stereotactic radiosurgery (SRS) are the available standard treatments (5). The estimation for prognosis of nervous system neoplasms is a challenging issue and remains rather poor, particularly for the patients with metastatic lesions. The median survival rate in patients with and without treatment is as low as only 3-21 and 1-2 months, respectively $(3,7)$. Among primary brain tumors, glioblastomas are the most fatal; These patients often do not survive beyond few months despite optimal multimodal treatments (8). Recent high throughput molecular and genomic technologies have revealed high molecular heterogeneity among phenotype of glioblastoma. This heterogeneity is responsible for treatment failure and poor prognosis $(9,10)$. Regarding the noninvasive nature of neuroimaging modalities, identifying of a prognostic imaging indicator correlated with histopathologic grades of the tumor, molecular characteristics, or clinical outcome of the disease is of vital value in disease prognostication and improve the patient care as well (11). PET imaging using novel radiotracers show promise in tumor grading and molecular characterization through visualizing various molecular and functional properties in tumor lesions $(5,11,12)$. This application of PET imaging is discussed in the current review.

\section{Role of neuroimaging in brain tumors}

Neuroimaging plays a pivotal role in various aspects of brain neoplasms evaluation during the clinical course of the disease. These include precise anatomic localization, tumor grading, delineation of tumor extent and margins for pre-surgical or radiotherapeutic planning, determination of ideal site for biopsy, identifying the involvement of vital structures, early response assessment, discrimination of tumor recurrence from reactive changes after therapy and prognostication. However, the definitive diagnosis is still made upon tissue biopsies $(5,11)$. Presently, magnetic resonance imaging (MRI) with gadolinium contrast agent is used as the standard method of choice in the practice of brain tumors. MRI enables visualizing anatomic details in the routine clinical setting (5). Perfusion-weighted MR measures the blood flow parameters; magnetic resonance spectroscopy (MRS) measures the concentration of metabolites within tissues; and diffusion tensor imaging (DTI) assess brain tumor by mapping the white matter (11,13). Functional MR imaging (fMRI) using the Blood Oxygen Level Dependent (BOLD) technique provides functional map for each patient facilitates the surgical planning and averts the unsolicited collateral damage to vital brain regions during surgery and radiotherapy (5).

\section{PET and brain tumors}

Many PET tracers have been produced for imaging in oncology, with different physiochemical identities, biologic targets, and labeled radionuclides. Among these, merely a few tracers have been employed for the imaging of brain tumor in humans. Since more than three decades ago, PET modality has been utilized for brain tumor imaging in both clinical practice and research setting. The glucose analog 2-deoxy-2-[fluorine-18] fluoro-D-glucose $\left({ }^{18} \mathrm{~F}\right.$-FDG) is the standard molecular imaging agent applied for brain lesions in the routine practice. In clinical neuro-oncology, ${ }^{18} \mathrm{~F}$-FDG has been utilized mainly to differentiate the tumor recurrence from radiation-induced changes. Novel PET offers biologic information not visible via ${ }^{18} \mathrm{~F}$-FDG PET 
modality or contrast-enhanced MRI alone.

\section{Role of PET imaging in the delineation of tumor volume}

Although the delineation of entire tumor volume is crucial in the diagnostic evaluations and pre-surgical resection or pre-radiotherapy planning, proper tumor delineation in the routine clinical practice remains a challenge. The reason is that the function of Blood-Brain-Barrier (BBB) that keeps contrast agents (CT or MR) outside of the brain parenchyma. Brain tumors, those which have intact BBB, such as low-grade gliomas do not enhance following the injection of intravenous CT or MR contrast agent, while high-grade glioma tumors usually have impaired BBBs and are enhanced with contrast agent. However, several high-grade tumors may not enhance with contrast agent, and even there are often non-enhancing regions within enhanced tumors in cases of glioblastomas (5). On the other hand, imaging of extra-axial lesions, which located outside of the brain, and metastases that interrupt BBB integrity do not suffer these issues.

Many of PET tracers can pass through the BBB owing to be the substrate of BBB transporters. Only those molecules or compounds can cross the BBB which are substrate for biological transporters located on the luminal surface of the BBB (5). Although the ${ }^{18} \mathrm{~F}-\mathrm{FDG}$ PET is a standard modality in oncologic imaging, this tracer suffer from its relative lack of specificity and high background uptake in healthy brain tissue that could lead to false negative or false positive results in lesions less than $5 \mathrm{~mm}$ (11). Two strategies have been developed to improve ${ }^{18} \mathrm{~F}$-FDG PET modality: (I) coregistration of ${ }^{18} \mathrm{~F}$-FDG $\mathrm{PET}$ with $\mathrm{MR}$ imaging in which MR imaging enables us to outline region of interest (ROI) accurately and any enhanced ${ }^{18} \mathrm{~F}$-FDG uptake represents an active process, and (II) delayed image acquisition, that is based on the lower rate constant of FDG-6-phosphate degradation at prolonged period of time in the tumor lesion compared with the healthy brain tissue, in which ${ }^{18} \mathrm{~F}-\mathrm{FDG}$ PET imaging is performed at an extended time interval between injection and PET imaging (6).

Recently, novel PET-tracers have been developed which have demonstrated better results than ${ }^{18} \mathrm{~F}$-FDG PET imaging $(5,6)$. Among them, radiolabeled amino acids have shown promising potential in accurate tumor volume delineation, active metabolic volume, and better results in pre-surgical or pre-radiotherapy planning $(5,6,14)$. An investigation revealed that the utility of hybrid imaging through fusing amino acid PET (or SPECT) with MR (or CT) imaging in the reirradiation planning of recurrent high-grade gliomas significantly improved survival time compared with CT or MR imaging alone (14). Investigators recruited 44 participants with recurrent high-grade gliomas after previous surgical resection and adjuvant conventional radiotherapy \pm chemotherapy (temozolomide). For treatment planning of fractionated stereotactic radiotherapy (FSRT), the gross tumor extent was delineated using hybrid imaging modality through fusing ${ }^{123}$ I-methyl-tyrosine (IMT) single-photon computed emission tomography (SPECT) or ${ }^{11} \mathrm{C}$-methionine positron emission tomography (METPET) with computed tomography (CT) or MRI in $82 \%$ of the subjects and by CT/T1gadolinium-MRI image fusion in $18 \%$ of them (Figures 1,2). Results showed that treatment designing using fused PET (SPECT)/MR (CT) imaging was correlated with the improvement of survival compared to treatment designation based on CT/MRI alone.

\section{Brain tumor grading and molecular characterization using PET imaging}

Accurate tumor grading is an immediate step in the evaluation and management of brain tumors. Investigators have been focusing on the concept of "non-invasive tumor grading", to identify a grading imaging marker which is capable to correlate with the histological grading of tumor tissues (11). ${ }^{18} \mathrm{~F}$-FDG uptake is usually higher in high grade glioma (WHO grade III and IV) than low grade tumors (WHO grade II) (15). The usefulness of ${ }^{18}$ F-FDG PET for grading of glioma tumors is hindered by the low tumor-tobackground ratio due to physiologically enhanced glucose metabolism of cortical and subcortical component in the brain (15). In the other studies, ${ }^{18} \mathrm{~F}$-FDG-PET failed to be correlated with ki-67 index, which is a histological indicator of cell proliferation $(16,17)$.

An increased uptake in the fluorothymidine (FLT) PET imaging are shown in high grade tumors as well (18-25). Collet and colleagues (18) demonstrated that the FLTPET imaging is superior to several advanced MR imaging modalities including both diffusion and perfusion weighted MR imaging and even MRS in noninvasive tumor grading. A total of thirty-nine patients with spread gliomas whose tumors were pathologically proven to be grade II, III, or IV were included. They were investigated using conventional MRI and perfusion, diffusion, and proton MR spectroscopy (1H-MRS) and ${ }^{18} \mathrm{~F}$-FLT-PET imaging prior to surgery. Apparent diffusion coefficient (ADC), relative cerebral 

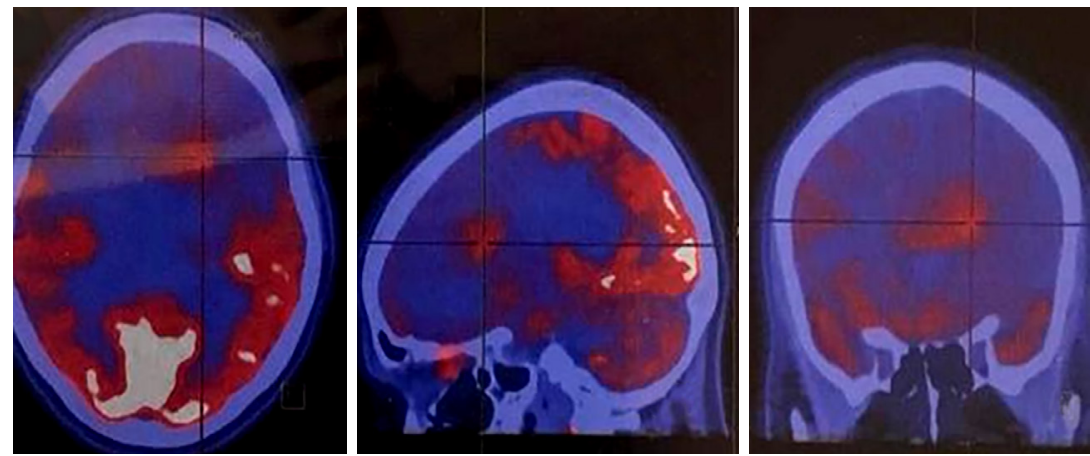

Figure $1{ }^{18} \mathrm{~F}$-FDG PET/CT scan of high-grade astrocytoma. There is asymmetry increased uptake in the left frontoparietal parasagittal region. ${ }^{68} \mathrm{Ga}$-DOTATATE PET/CT scan showed no abnormal activity (not shown).
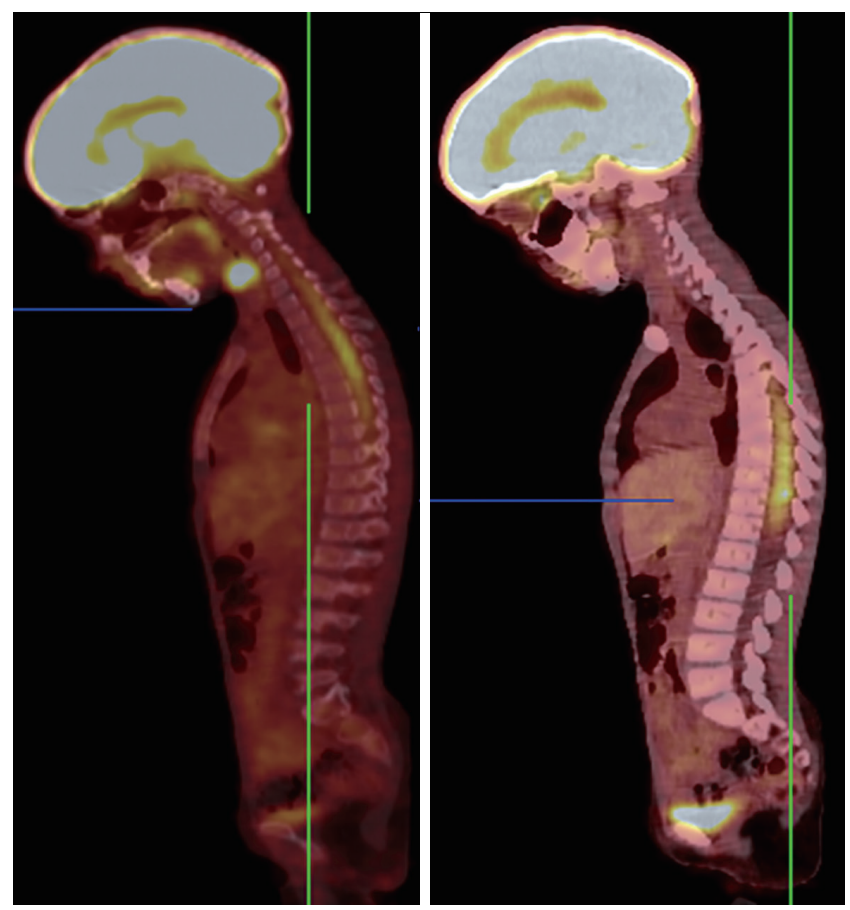

Figure $2{ }^{18} \mathrm{~F}$-FDG PET/CT scan of a patient with spinal cord medulloblastoma. PET/CT images demonstrated diffuse enhanced FDG uptake in spinal cord at level of T5-T11.

blood volume (rCBV), Cho/Cr, NAA/Cr Cho/NAA and FLT SUV were compared among grades. Results revealed that the mean value of ${ }^{18} \mathrm{~F}$-FLT uptake $\mathrm{FLT}_{\text {mean }}(\mathrm{R} 2=0.36)$ and $\mathrm{FLT}_{\text {max }}(\mathrm{R} 2=0.5)$ were the best indicators that correlate with tumor grades, even superior to MRS especially regarding differentiation between doubtful grade III and IV tumors.

Of the remarkable progress in advanced molecular profiling and genomic characterization in brain tumors, particularly in gliomas, was the revision of the classification and management of gliomas toward molecular profiling and genomic characterization by WHO in 2016 (1). Vomacka and colleagues (26) have intended to ascertain a readerindependent glioma detection and characterization of aggressive sub-component through a voxel-wise analysis of diagnostically-related static and kinetic ${ }^{18} \mathrm{~F}$-FET PET values. 162 subjects with newly diagnosed gliomas were enrolled and were separated into groups upon histologic, genetic, and molecular characteristics. They measured the biological tumor volume (BTV), and also for every enclosed voxel, the late slope $\left(\mathrm{Slope}_{15-40 \mathrm{~min}}\right)$, the timeto-peak (TTP), and the tumor-to-background ratios $\left(\mathrm{TBR}_{5-15 \text { min }}, \mathrm{TBR}_{20-40 \mathrm{~min}}\right)$ were calculated. The percentage portion of these parameter inside the BTV was assessed with the percentage volume fractions (PVFs) and cumulated percentage volume histograms (PVHs). The potential of these voxel-based parameters to differentiate among molecular genetics properties and histologic grades was appraised and compared with volume-of-interest (VOI)based values. In the voxel-wise analysis, high grade glioma tumors, WHO grade III and IV, and IDH-wild type gliomas predominantly contained high proportion of voxels with negative slope, high early peak, and high TBR. It also has been shown that PVHs with TTP $<20$ min p.i., Slope $_{15-40 \text { min }}$ $<0 \mathrm{SUV} / \mathrm{h}$, and $\mathrm{TBR}_{5-15 \text { min }}$ and $\mathrm{TBR}_{20-40 \text { min }}>2$ yielded the most significant difference between tumor grades. Moreover, voxel-based parameters compared with VOIindices showed better accuracy in both tumor grading and IDH mutation status. The researchers provided an automated and reader-independent method for voxelwise ${ }^{18} \mathrm{~F}$-FET PET glioma analysis that has potential to 
differentiate among WHO grades of glioma tumors and between IDH mutations subtypes. Finally, they concluded that the designated method potentially offers a rapid determination of aggressive subdivision of tumor within the $\mathrm{BTV}$, and parametric 3D ${ }^{18} \mathrm{~F}$-FET PET information may contribute to guiding biopsy acquisition in heterogeneousnature glioma tumors.

\section{Promises of new artificial amino acids in non-invasive tumor grading}

So far, many amino acid transporters have been detected at molecular level and have been described in the human body. Natural amino acids is absorbed via amino acid transport systems that important systems include the A, L, and alanineserine-cysteine (ASC) amino acid transport systems (27). L amino acid transport System are $\mathrm{Na}+$-independent, and the principal transport pathway for phenylalanine, tyrosine, methionine, and their analogs, but, System A and ASC systems are $\mathrm{Na}$--dependent (27). However, the literature is scarce regarding the application of radiolabeled amino acids transported through an amino acid transporter system in the cancer management investigations.

[N-methyl-C-11]-methylaminoisobutyric acid (MeAIB) is a synthetic amino acid transported through system A neutral amino acid transports and is recently used for brain tumors (28). In 2018, Nishii and colleagues (28) studied the ability of MeAIB for noninvasively differentiating high-grade from low-grade gliomas in clinical setting and compared it with the ability of [S-methyl-C-11]-Lmethionine (MET)-PET. Thirty-one individuals (male: 16, female: 15) participated in the study. They were separated into two groups: Group A comprised of individuals who were diagnosed with benign lesion such as low-grade astrocytoma tumors, grade II or less, or other low-grade astrocytomas $(n=12)$. Group B including patient with malignant brain tumors such as glioblastoma multiforme $(\mathrm{GBM})$, recurrent $\mathrm{GBM}$, or anaplastic astrocytoma $(\mathrm{n}=19)$. All of the participants underwent MeAIB-PET and METPET imaging. The results showed that the SUVmax values were $1.20 \pm 1.29$ for the group A patients and $2.94 \pm 1.22$ for group B patients on MeAIB-PET imaging. The tumor-tocontralateral normal brain tissue (T/N ratio) was $3.77 \pm 2.39$ in group A patients and $16.83 \pm 2.39$ in group B patients. In MET-PET, the SUVmax was 3.01 \pm 0.94 in group A patients and $4.72 \pm 1.61$ in group $B$ patients, and the $T / N$ ratio was $2.64 \pm 1.40$ for group A and $3.21 \pm 1.14$ for group B. The investigators concluded that the MeAIB-PET imaging yielded higher accuracy than the MET-PET imaging in distinguishing low-grade from high-grade brain tumors.

\section{Assessment of response to treatment using hybrid imaging}

Regarding the highly aggressive and heterogeneous nature of brain tumors, especially GBM as the most common and fatal of those, earlier response assessment is vital in the management of brain tumors. At present, current clinical practice relies upon conventional imaging to appraise the response. It remains a challenge to determine the treatment efficacy due to the lack of accuracy of conventional modalities in differentiating between pseudoprogression (PsP) including treatment-induced changes, and true tumor progression (tumor growth or recurrence) $(6,11)$. In recent years, investigators have made great efforts to overcome the challenges through utilizing molecular imaging like PET imaging, which uses a vast range of tracers with different properties, and with combined anatomical and functional modalities e.g., PET/MR (11,29-32). FDG-PET, the current standard PET agent, utilization in differentiating between pseudo- and true progression has shown variable results (6). In addition, high background uptake may limit its ability regarding small lesion detection (11). Recently, new PET tracers have demonstrated better results than the traditional FDG-PET in differentiating PsP and true progression of tumors $(33,34)$. Gao et al. evaluated the diagnostic precision of $11 \mathrm{C}$-choline PET in differentiating PsP from true tumor progression through a meta-analysis. With a total of 118 patients (134 scans), the pooled sensitivity and specificity were 0.87 (95\% CI: $0.78,0.93)$ and 0.820 (95\% CI: $0.69,0.91$ ), respectively. They conclude that ${ }^{11} \mathrm{C}$-choline has the potential to differentiate PsP and true progression of tumors (35). Some studies have further suggested that $\mathrm{O}-\left[2-(18 \mathrm{~F})\right.$ fluoroethyl]-L-tyrosine $\left({ }^{18} \mathrm{FET}\right)$ PET can potentially solve this problem $(31,36)$. Galldiks and colleague studied the detection of pseudoprogression in glioblastoma patients using ${ }^{18} \mathrm{~F}$-FET-PET. They investigated a total of 22 patients with new contrastenhanced (CE) areas or lesions that enhanced with contrast $(>25 \%)$ on MR imaging during the first 12 weeks after finishing the course of therapy. Patients underwent ${ }^{18}$ F-FET-PET as well to differentiate PsP from true progression. They showed that the best ${ }^{18} \mathrm{~F}$-FET $\mathrm{TBR}_{\max }$ cut-off value for diagnosis of PsP was 2.3 (sensitivity $100 \%$, specificity $91 \%$, accuracy $96 \%$ ) (31). Lohmann et al. explored the potential of combined FET-PET/MRI 

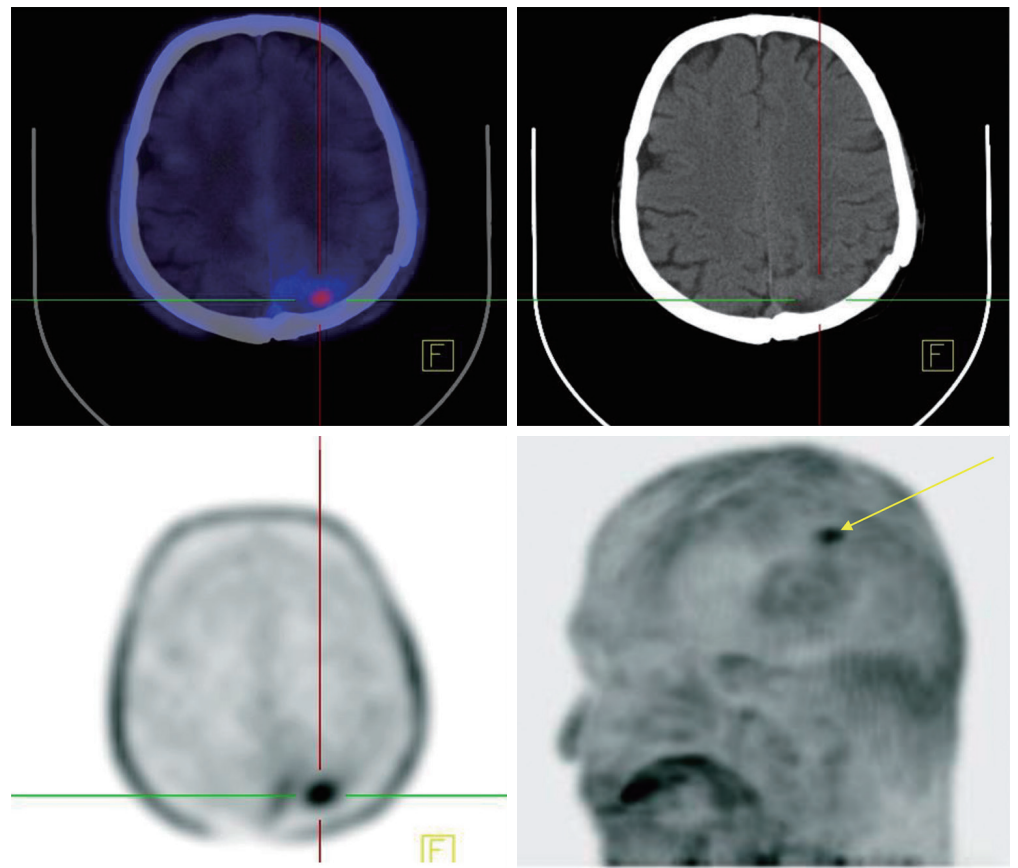

Figure $3{ }^{18} \mathrm{~F}$-FET PET/CT scan representing tumor recurrence in the posterior left occipital lobe (arrow).

in distinguishing of $\mathrm{PsP}$ from true progression in the brain metastasis. A total of 52 patients who showed new or progressive contrast-enhancement lesions on MR imaging after ending the radiotherapy underwent the FET-PET as well. The results showed that the CE-MRI had an accuracy of $81 \%$ (sensitivity, $67 \%$; specificity, $90 \%$ ) in PsP diagnosis. The diagnosis accuracy for FET-PET was $83 \%$ (sensitivity, $88 \%$; specificity, $75 \%$ ). They combined the two modalities and achieved a diagnostic accuracy of $89 \%$ (sensitivity, $85 \%$; specificity, $96 \%$ ). Thus, it may be the right time to shift from conventional imaging to the fused anatomical/ molecular imaging approach which uses new agents to improve diagnostic accuracy and provides further insight into the tumor characteristics (Figure 3).

\section{Application of novel PET tracers in brain tumors}

\section{Somatostatin receptor (SSTR) imaging in brain neoplasms other than neuroendocrine tumors (NETS)}

Somatostatin is a neuropeptide released by endocrine cells, neurons, and immune cells which its functions include neuroregulation (sensory, motor, and cognition) and cell growth inhibition by paracrine and autocrine pathways $(37,38)$. Somatostatin works through a group of transmembrane $\mathrm{G}$ protein-coupled receptors that enter the cell following binding to specific ligands (39). So far, six subtypes of somatostatin receptors (SSTRs) are discovered including SSTR 1, 2A, 2B, 3, 4, and 5. A broad spectrum of brain tumors express different subtypes of SSTR on their surface including frequent primary brain tumors e.g., gliomas and meningiomas, pediatric brain tumors (medulloblastomas), pituitary adenomas, and supratentorial primitive neuroendocrine tumors (PNETs) (40-43). Dutour and colleague (44) have shown that gliomas and meningiomas, as the first and second most common primary brain tumors, are positive for different subtypes of SSTR. It was demonstrated that although all of them have at least one SSTR subtype, many of these harboring multiple SSTR subtypes. They provided proofs on detecting SSTRs in both tumors and their surrounding tissues, particularly blood vessels. Concerning gliomas, low-grade gliomas (WHO grade I, II) depicted higher expression levels compared to high-grade gliomas (WHO grade III, IV) (45). To date, three ${ }^{68} \mathrm{Ga}$-DOTA peptides are developed for clinical imaging comprised of ${ }^{68} \mathrm{Ga}-\mathrm{DOTA}$-TOC, ${ }^{68} \mathrm{Ga}$-DOTANOC, and ${ }^{68} \mathrm{Ga}$-DOTA-TATE. The shared property of these ${ }^{68} \mathrm{Ga}$-DOTA peptides is the ability in binding to SSTR2, and they differ with regard to their SSTR subtype affinity and profile (Figures 4,5). 

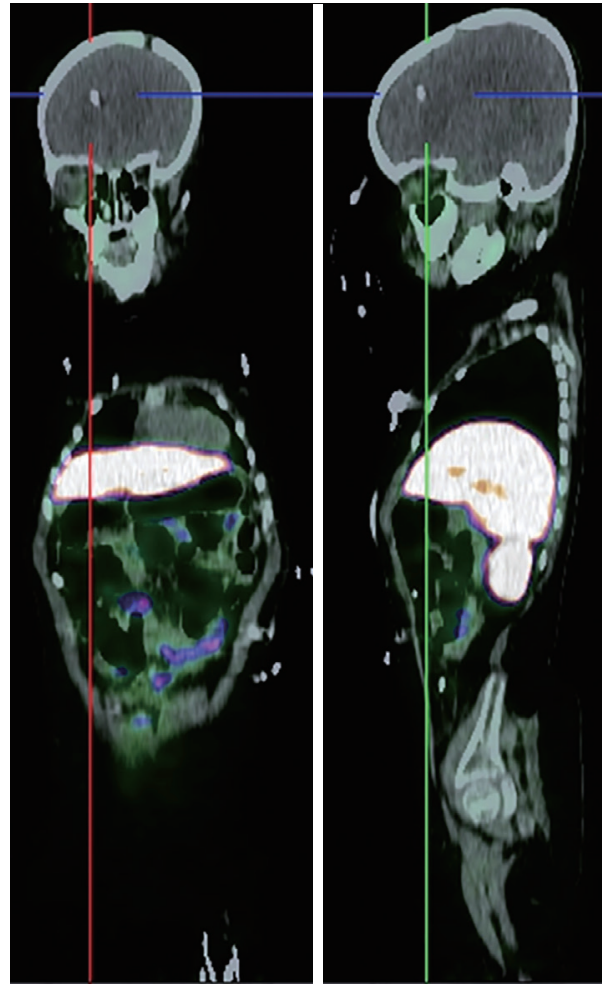

Figure $4{ }^{68} \mathrm{Ga}$-DOTATATE PET/CT scan of glioma. There is radiotracer uptake in a large mass in an ill-defined mass with increased uptake with SUVmax $=10.48$.

\section{Ga-DOTA-peptide in meningiomas}

Meningioma is the second most frequent primary brain tumor and is benign in $90 \%$ of cases. The current standard imaging modality in diagnosis is conventional MR imaging (43). Although the SSTR receptors are positive in the healthy leptomeningeal cell, the meningioma cells express higher levels of them (46,47). Afshar-Oromieh et al. (48) aimed to compare the ability in detecting meningioma lesions between ${ }^{68} \mathrm{Ga}$-DOTA-peptide PET/ CT and contrast-enhanced MRI (CE-MRI) within a total of 134 patient. They revealed that ${ }^{68} \mathrm{Ga}-68 \mathrm{Ga}$-DOTA-peptide PET/CT has higher accuracy compared to CE-MRI in meningioma detection (190 vs. 171). The investigators concluded that ${ }^{68} \mathrm{Ga}$-DOTA-peptide PET/CT could play a complementary role with MRI in cases where MRI finding has failed in definitive diagnosis or lake of feasibility for biopsy. Another promising potential of ${ }^{68} \mathrm{Ga}$-DOTA-peptide PET/CT was reported by Milker-Zabel and colleagues (49) through a study that showed target volume delineation using ${ }^{68} \mathrm{Ga}$-DOTA-PET/CT and MRI in comparison to
CT or MRI alone has changed the radiotherapy planning in $73 \%$ of 26 patients. Gehler et al. (50) reported similar results as well. One example of this modality in meningioma is illustrated in Figure 6.

\section{Ga-DOTA peptide in medulloblastomas}

Medulloblastoma is the most prevalent type of the brain tumor among pediatrics, with the highest incidence between the ages of 3 to 9 years $(43,51)$. The primary treatment is craniospinal radiotherapy with significant rates of cure but together with severe neurocognitive decline (43). It has been revealed that medulloblastomas express high levels of SSTR receptors, with highest expressed subtype being SSTRs 2 and 3 (45,52). Up to our knowledge, no study has been published yet on medulloblastoma evaluation using ${ }^{68} \mathrm{Ga}$-DOTA-peptide PET/CT. It is possible that ${ }^{68} \mathrm{Ga}-$ DOTA-peptide PET/CT can have a valuable role in the management of medulloblastomas.

\section{Role of prostate-specific membrane antigen (PSMA) in brain tumors imaging}

PSMA is a type II transmembrane glycoprotein which is overexpressed in prostate cancer and its expression levels are positively associated with tumor grade and stage (53). Also, PSMA expression is increased with conditions like hormone resistance, metastatic lesions, and anaplastic properties in tumor (54). Although PSMA was found in prostate glands for the first time but further histologic studies revealed it could be found in other healthy tissue as well, including salivary glands, lacrimal glands, duodenal epithelium, proximal tube cell of the kidney $(55,56)$. Of note, it has been detected in in healthy tissues (as mentioned earlier), benign conditions (e.g., meningiomas in the brain), and malignant tumors both prostatic-originated and nonprostatic-originated tumors, therefore questioning the sensitivity and specificity of PSMA as a specific prostatic tracer $(53,57,58)$. Since the development of ${ }^{68} \mathrm{Ga}$-labeled PSMA, a PSMA-targeted small molecule labeled with ${ }^{68} \mathrm{Ga}$, investigators are equipped not only to visualize the prostate cancer throughout the human body, but also to use it as a therapeutic target (59-61). Although PSMA has been developed to assess the management of prostate cancer, the expression of PSMA has been seen in different nonprostatic malignant and nonmalignant conditions causing to probable difficulties in the analysis of PSMA-targeted studies. On the other hand, this limitation may indicate favorable in imaging and therapeutic agent for other nonprostatic 

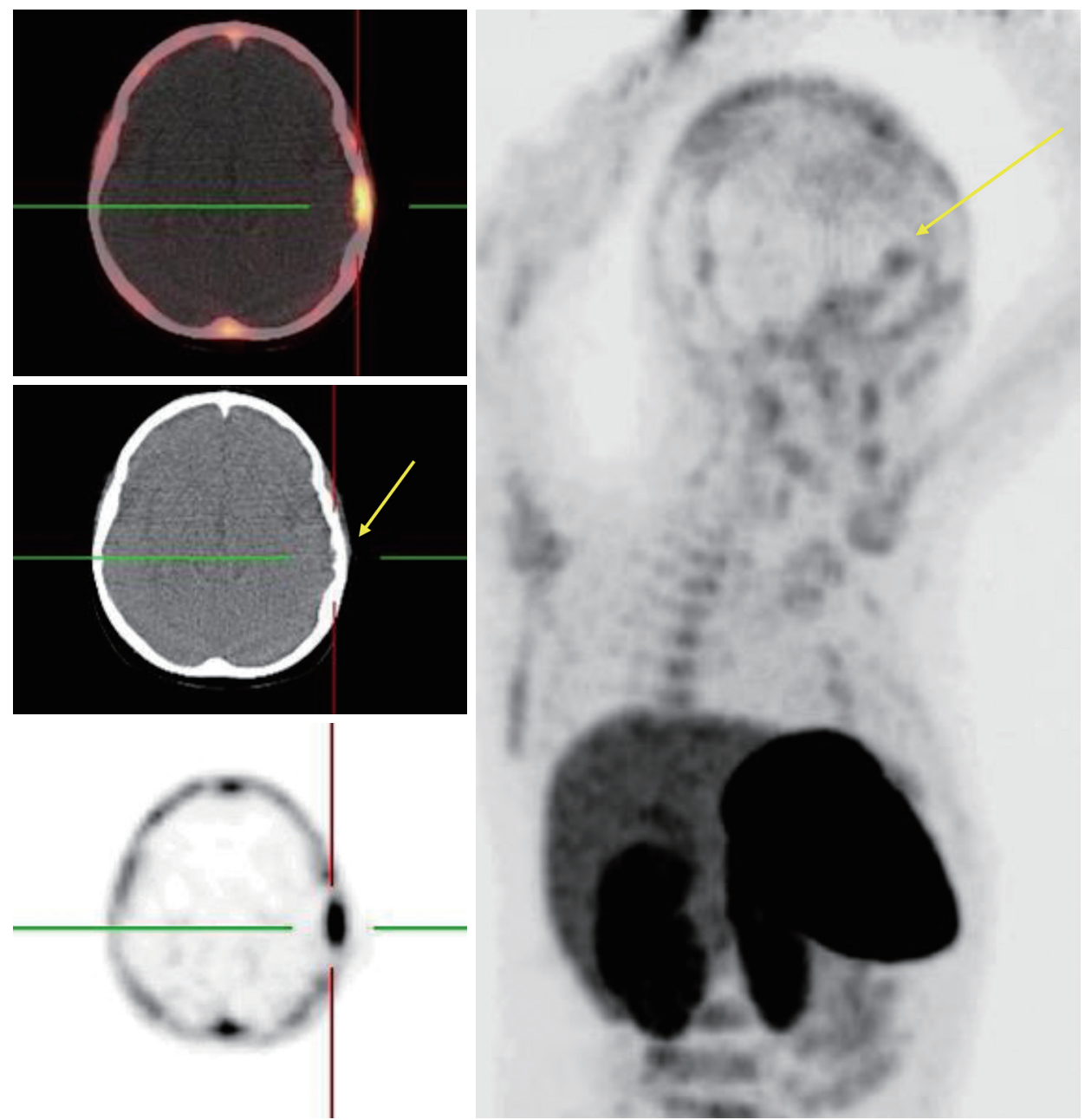

Figure $5{ }^{68} \mathrm{Ga}$-DOTATATE PET/CT scan of neuroblastoma. There is focal uptake in the skull representing bony invasion (arrow).

conditions $(53,56) .{ }^{68} \mathrm{Ga}$ labeled PSMA is increasingly incorporated in the routine clinical assessment of patients affected by prostate cancer. Whole-body ${ }^{68} \mathrm{Ga}$-PSMA scan can be useful in the initial staging of intermediate or high risk prostate cancers and in restaging after biochemical clue of tumor recurrence $(62,63)$ (Figure 7 ).

Healthy brain tissue does not show any PSMA uptake which makes it a promising tracer in detecting metastasis of prostatic cancer to the brain, but studies reported new connections between PSMA uptake and nervous system tumors other than prostatic metastasis $(57,59)$. Up to present, several studies have reported PSMA expression in few nervous system tumors (53) including meningiomas, schwannomas, peripheral nerve sheath tumors as well as other benign conditions such as sympathetic chain ganglia (58,64-71). So far, two prostatic cancer patients are reported who showed incidental increased uptake of ${ }^{68} \mathrm{Ga}$-PSMA-11 (PET/CT) with corresponding MRI imaging consistent with meningiomas $(58,64)$. PSMA-radiotracer uptake in other neurogenic conditions occurs in scenarios similar to meningiomas. Regarding the low incidence of prostatic cancer metastasis to the brain and lack of tracer uptake by the healthy brain, it seems reasonable to consider further imaging or even examining tissue biopsies to confirm the diagnosis. These recent studies gave us better insights into the PSMA expressions status throughout the body and, with this in mind, increased uptake of PSMA-targeted tracers in the brain of prostatic cancer subjects may require further 

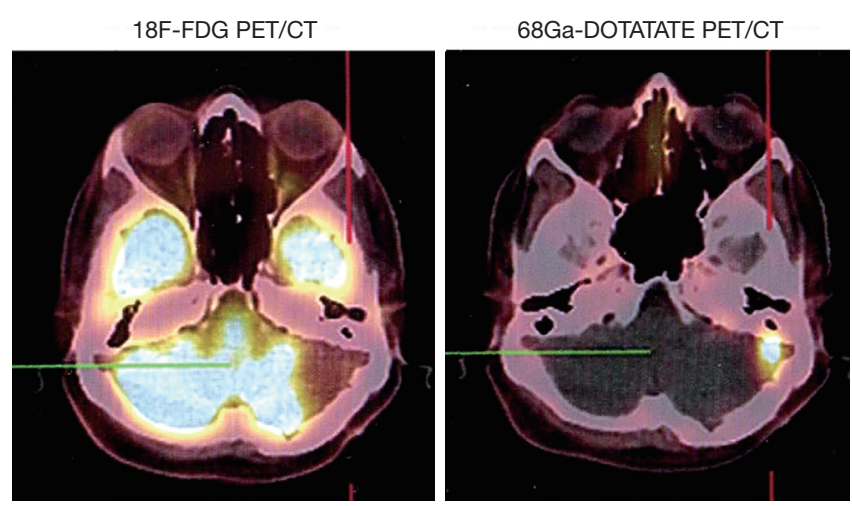

Figure $6{ }^{68} \mathrm{Ga}$-DOTATATE PET/CT scan of meningioma. An intense focus of uptake is detected in the left vermis of the left cerebellum representing remnant/recurrence tumor. Absent uptake in the same region on ${ }^{18} \mathrm{~F}-\mathrm{FDG}$ PET/CT.

imaging to establish the diagnosis.

\section{Imaging-based precision medicine using PET in the patients with the brain tumors}

National Institutes of Health (NIH) have described the precision medicine as "an emerging approach for disease treatment and prevention that takes into account individual variability in genes, environment, and lifestyle for each person" (72). This requires clinicians and investigators to tailor specified treatments for each of the affected individuals through targeting cancer cells with less damage to the normal tissues $(72,73)$. Medicine is facing a significant paradigm shift from reactive to a proactive manner in all aspects of clinical practice and healthcare through the development of precision medicine and high throughput molecular technologies $(12,74)$. Molecular imaging has the capacity to play a substantial role in the development and future application of precision medicine at the front line of cancer investigations, especially neuro-oncology (72). ImmunoPET, a PET imaging technique that takes advantage of antibodies as targeting probes, has shown promising results in noninvasively assessing the expression levels of tumorrelated antigens in vivo $(75,76)$. In some reported cases, investigators have suggested that Immuno-PET is superior to magnetic resonance imaging (MRI) and ${ }^{18} \mathrm{~F}-\mathrm{FDG}$ PET regarding the sensitivity and specificity of cancer assessment $(77,78)$. In one preclinical study, CD146 expression was explored using both tissue studies and longitudinal Immuno-PET for glioblastoma in determining sensitivity and specificity of diagnosis and therapeutic potentials (75). The investigators generated an innovative ${ }^{89} \mathrm{Zr}$-labeled monoclonal antibody (mAb), ${ }^{89} \mathrm{Zr}$-Df-YY146, for targeting and measurement of CD146 expression in a mouse model of glioblastoma. They performed immunofluorescence microscopy, flow cytometry, and Western blot with two cell lines of glioblastoma, U87MG and U251, as in vitro assays to define their expression level of CD146. Also, YY146's CD146-binding affinities in comparison to that of Df-YY146 were assessed using flow cytometry. ${ }^{89} \mathrm{Zr}$-DfYY146 ability in targeting CD146 was explored through sequential PET imaging in vivo. In addition, ex-vivo biodistribution, CD146 blocking and histological assessment were performed to verify ${ }^{89} \mathrm{Zr}$-Df-YY146 specificity and PET data validation as well. In vitro assays demonstrated increased expression level of CD146 in U87MG cells, but negligible level CD146 in U251 cells. Flow cytometry showed no difference in affinity between Df-YY146 and YY146. ${ }^{89} \mathrm{Zr}$-labelled-Df-YY146 proceeded with outstanding yield $(\sim 80 \%)$, radiochemical purity $(>95 \%)$, and specific activity $(\sim 4 \mathrm{GBq} / \mu \mathrm{mol})$. Longitudinal PET showed considerable and permanent ${ }^{89} \mathrm{Zr}$-Df-YY146 uptake in mice harboring U87MG tumor cells, with significantly lesser in CD146-negative U251 lesions at 48 hours after tracer administration. Tracer uptake in U87MG tumor cells was effectively hindered in a competitive inhibition assays that supported the ${ }^{89} \mathrm{Zr}-\mathrm{Df}-\mathrm{YY} 146$ specificity. Eventually, ex-vivo biodistribution confirmed the accuracy of PET values and histological studies strongly correlated tracer uptake with insitu expression level of CD146. Given the significant, specific and persistent uptake of ${ }^{89} \mathrm{Zr}$-Df-YY146 by brain tumors, it may be interesting to perform non-invasive PET scan to evaluate CD146 expression which may serve as a novel tool for intervention guidance and response assessment to the treatment. Therefore, PET imaging, especially ImmunoPET modality in combination with quantitative analytical software tools, may play a key role in diagnosis, molecular profiling, treatment, and response assessment through accurately visualizing different molecular characteristics and pathophysiologic processes $(5,6,72,75,77,78)$.

\section{Conclusion and prospective}

PET imaging with a vast range of radiotracers has the ability to visualize versatile molecular properties and pathophysiological processes within the human body. Given the present paradigm shift in the medicine from reactive to proactive approaches, molecular characterization and 


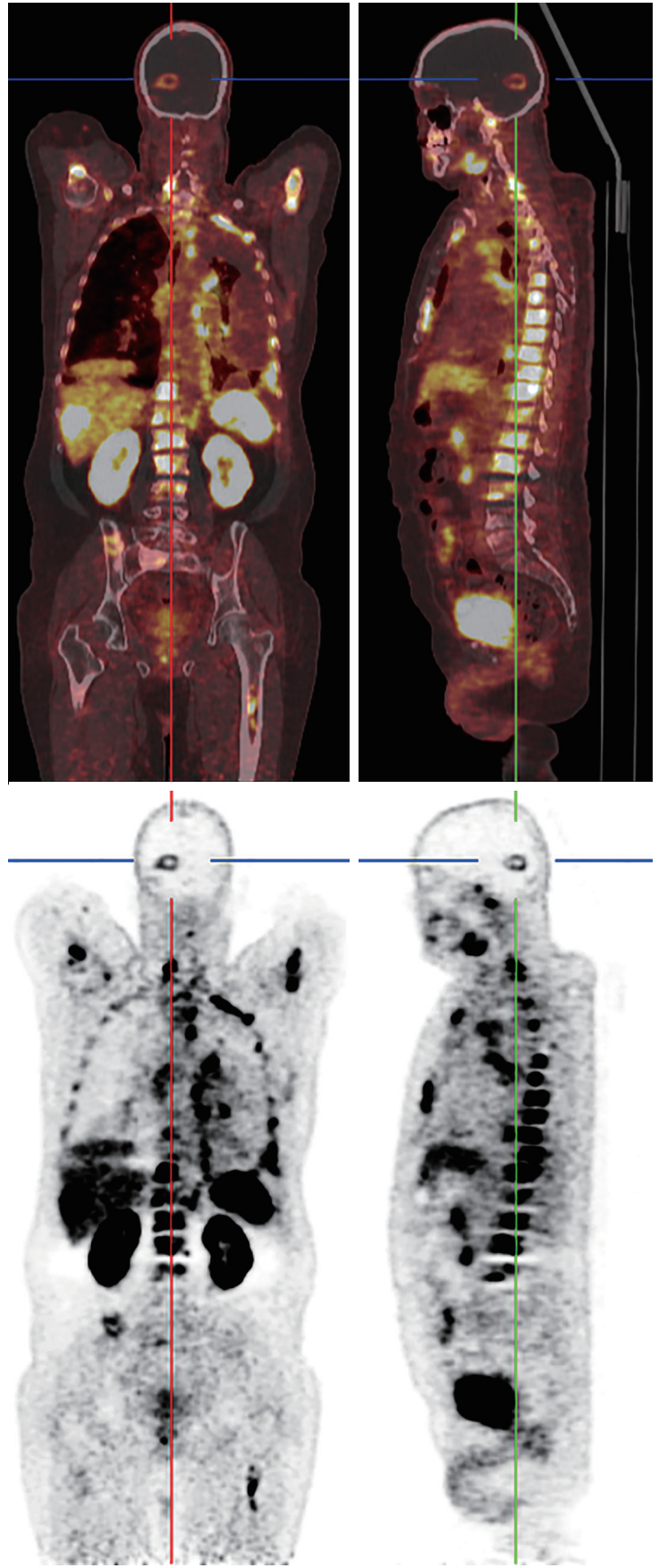

Figure 7 68Ga-PSMA PET/CT scan of prostate cancer. In addition to widespread metastases, there is focal uptake in the brain.

localization are considered as a mainstay of it. PET tracers can play an eminent role in providing tailored treatment through carrying radiolabeled payloads to a target-specified site. PET imaging has the potential to be considered as an important complementary modality in addition to MRI for improving the accuracy in various aspects of brain tumor management.

\section{Acknowledgments}

None.

\section{Footnote}

Conflicts of Interest: The authors have no conflicts of interest to declare.

\section{References}

1. Louis DN, Perry A, Reifenberger G, Von Deimling A, Figarella-Branger D, Cavenee WK, Ohgaki H, Wiestler OD, Kleihues P, Ellison DW. The 2016 World Health Organization classification of tumors of the central nervous system: a summary. Acta Neuropathol 2016;131:803-20.

2. Siegel RL, Miller KD, Jemal A. Cancer statistics, 2018. CA Cancer J Clin 2018;68:7-30.

3. Soffietti R, Ducati A, Rudà R. Brain metastases. Handbook of Clinical Neurology. Elsevier; 2012. p. 747-55.

4. Nayak L, Lee EQ, Wen PY. Epidemiology of brain metastases. Curr Oncol Rep 2012;14:48-54.

5. Sharma A, McConathy J. Overview of PET tracers for brain tumor imaging. PET Clin 2013;8:129-46.

6. Basu S, Alavi A. Molecular imaging (PET) of brain tumors. Neuroimaging Clin N Am 2009;19:625-46.

7. Sperduto PW, Chao ST, Sneed PK, Luo X, Suh J, Roberge D, Bhatt A, Jensen AW, Brown PD, Shih H. Diagnosisspecific prognostic factors, indexes, and treatment outcomes for patients with newly diagnosed brain metastases: a multi-institutional analysis of 4,259 patients. Int J Radiat Oncol Biol Phys 2010;77:655-61.

8. Ostrom QT, Gittleman H, Fulop J, Liu M, Blanda R, Kromer C, Wolinsky Y, Kruchko C, Barnholtz-Sloan JS. CBTRUS statistical report: primary brain and central nervous system tumors diagnosed in the United States in 2008-2012. Neuro Oncol 2015;17 Suppl 4:iv1-62.

9. Mrugala MM. Advances and challenges in the treatment of glioblastoma: a clinician's perspective. Discov Med 2013;15:221-30.

10. Omuro A, DeAngelis LM. Glioblastoma and other malignant gliomas: a clinical review. JAMA 2013;310:1842-50.

11. Nandu H, Wen PY, Huang RY. Imaging in neuro-oncology. 
Ther Adv Neurol Disord 2018;11:1756286418759865.

12. Assadi $M$, Nabipour I. The future of molecular imaging in paradigm shift from reactive to proactive $(\mathrm{P} 4)$ medicine: predictive, preventive, personalized and participatory. Nucl Med Commun 2014;35:1193-6.

13. Hayashida Y, Hirai T, Morishita S, Kitajima M, Murakami R, Korogi Y, Makino K, Nakamura H, Ikushima I, Yamura M. Diffusion-weighted imaging of metastatic brain tumors: comparison with histologic type and tumor cellularity. AJNR Am J Neuroradiol 2006;27:1419-25.

14. Grosu AL, Weber WA, Franz M, Stärk S, Piert M, Thamm R, Gumprecht H, Schwaiger M, Molls M, Nieder C. Reirradiation of recurrent high-grade gliomas using amino acid PET (SPECT)/CT/MRI image fusion to determine gross tumor volume for stereotactic fractionated radiotherapy. Int J Radiat Oncol Biol Phys 2005;63:511-9.

15. Albert NL, Weller M, Suchorska B, Galldiks N, Soffietti R, Kim MM, La Fougere C, Pope W, Law I, Arbizu J. Response Assessment in Neuro-Oncology working group and European Association for Neuro-Oncology recommendations for the clinical use of PET imaging in gliomas. Neuro Oncol 2016;18:1199-208.

16. Kubota K. From tumor biology to clinical PET: a review of positron emission tomography (PET) in oncology. Ann Nucl Med 2001;15:471-86.

17. Kim S, Chung JK, Im SH, Jeong JM, Lee DS, Kim DG, Jung HW, Lee MC. 11 C-methionine PET as a prognostic marker in patients with glioma: comparison with 18 F-FDG PET. Eur J Nucl Med Mol Imaging 2005;32:52-9.

18. Collet S, Valable S, Constans J, Lechapt-Zalcman E, Roussel S, Delcroix N, Abbas A, Ibazizene M, Bernaudin M, Barré L. [18F]-fluoro-l-thymidine PET and advanced MRI for preoperative grading of gliomas. Neuroimage Clin 2015;8:448-54.

19. Shiroishi MS, Castellazzi G, Boxerman JL, D'amore F, Essig M, Nguyen TB, Provenzale JM, Enterline DS, Anzalone N, Dörfler A. Principles of T2*-weighted dynamic susceptibility contrast MRI technique in brain tumor imaging. J Magn Reson Imaging 2015;41:296-313.

20. Morita N, Wang S, Chawla S, Poptani H, Melhem ER. Dynamic susceptibility contrast perfusion weighted imaging in grading of nonenhancing astrocytomas. J Magn Reson Imaging 2010;32:803-8.

21. Julià-Sapé M, Coronel I, Majós C, Candiota AP, Serrallonga M, Cos M, Aguilera C, Acebes JJ, Griffiths JR, Arús C. Prospective diagnostic performance evaluation of single-voxel 1H MRS for typing and grading of brain tumours. NMR Biomed 2012;25:661-73.
22. Tate AR, Underwood J, Acosta DM, Julià-Sapé M, Majós C, Moreno-Torres À, Howe FA, Van Der Graaf M, Lefournier V, Murphy MM. Development of a decision support system for diagnosis and grading of brain tumours using in vivo magnetic resonance single voxel spectra. NMR Biomed 2006;19:411-34.

23. Vicente J, Fuster-Garcia E, Tortajada S, García-Gómez JM, Davies N, Natarajan K, Wilson M, Grundy RG, Wesseling P, Monleón D. Accurate classification of childhood brain tumours by in vivo $1 \mathrm{H}$ MRS-a multicentre study. Eur J Cancer 2013;49:658-67.

24. García-Gómez JM, Luts J, Julià-Sapé $M$, Krooshof $P$, Tortajada S, Robledo JV, Melssen W, Fuster-García E, Olier I, Postma G. Multiproject-multicenter evaluation of automatic brain tumor classification by magnetic resonance spectroscopy. MAGMA 2009;22:5-18.

25. Al-Okaili RN, Krejza J, Woo JH, Wolf RL, O'Rourke DM, Judy KD, Poptani H, Melhem ER. Intraaxial brain masses: MR imaging-based diagnostic strategy_initial experience. Radiology 2007;243:539-50.

26. Vomacka L, Unterrainer M, Holzgreve A, Mille E, Gosewisch A, Brosch J, Ziegler S, Suchorska B, Kreth FW, Tonn JC, Bartenstein P, Albert NL, Boning G. Voxel-wise analysis of dynamic (18)F-FET PET: a novel approach for non-invasive glioma characterisation. EJNMMI Res 2018;8:91.

27. Kagawa S, Nishii R, Higashi T, Yamauchi H, Ogawa E, Okudaira H, Kobayashi M, Yoshimoto M, Shikano N, Kawai K. Relationship between [14C] MeAIB uptake and amino acid transporter family gene expression levels or proliferative activity in a pilot study in human carcinoma cells: Comparison with $[3 \mathrm{H}]$ methionine uptake. Nucl Med Biol 2017;49:8-15.

28. Nishii R, Higashi T, Kagawa S, Arimoto M, Kishibe Y, Takahashi M, Yamada S, Saiki M, Arakawa Y, Yamauchi H. Differential Diagnosis between Low-Grade and HighGrade Astrocytoma Using System A Amino Acid Transport PET Imaging with C-11-MeAIB: A Comparison Study with C-11-Methionine PET Imaging. Contrast Media Mol Imaging 2018;2018:1292746.

29. Lohmann P, Kocher M, Ceccon G, Bauer EK, Stoffels G, Viswanathan S, Ruge MI, Neumaier B, Shah NJ, Fink GR. Combined FET PET/MRI radiomics differentiates radiation injury from recurrent brain metastasis. Neuroimage Clin 2018;20:537-42.

30. Kebir S, Khurshid Z, Gaertner FC, Essler M, Hattingen E, Fimmers R, Scheffler B, Herrlinger U, Bundschuh RA, Glas M. Unsupervised consensus cluster analysis 
of [18F]-fluoroethyl-L-tyrosine positron emission tomography identified textural features for the diagnosis of pseudoprogression in high-grade glioma. Oncotarget 2017;8:8294.

31. Galldiks N, Dunkl V, Stoffels G, Hutterer M, Rapp M, Sabel M, Reifenberger G, Kebir S, Dorn F, Blau T. Diagnosis of pseudoprogression in patients with glioblastoma using O-(2-[18 F] fluoroethyl)-L-tyrosine PET. Eur J Nucl Med Mol Imaging 2015;42:685-95.

32. Galldiks N, Stoffels G, Ruge MI, Rapp M, Sabel M, Reifenberger G, Erdem Z, Shah NJ, Fink GR, Coenen $\mathrm{HH}$. Role of O-(2-18F-fluoroethyl)-L-tyrosine PET as a diagnostic tool for detection of malignant progression in patients with low-grade glioma. J Nucl Med 2013;54:2046-54.

33. Chung J-K, Kim Y, Kim S-k, Lee Y, Paek S, Yeo J, Jeong J, Lee D, Jung H, Lee M. Usefulness of 11 C-methionine PET in the evaluation of brain lesions that are hypo-or isometabolic on 18 F-FDG PET. Eur J Nucl Med Mol Imaging 2002;29:176-82.

34. Li Z, Yu Y, Zhang H, Xu G, Chen L. A meta-analysis comparing 18F-FLT PET with 18F-FDG PET for assessment of brain tumor recurrence. Nucl Med Commun 2015;36:695-701.

35. Gao L, Xu W, Li T, Zheng J, Chen G. Accuracy of $11 \mathrm{C}$-choline positron emission tomography in differentiating glioma recurrence from radiation necrosis: A systematic review and meta-analysis. Medicine (Baltimore) 2018;97:e11556.

36. Langen K-J, Hamacher K, Weckesser M, Floeth F, Stoffels G, Bauer D, Coenen HH, Pauleit D. O-(2-[18F] fluoroethyl)-L-tyrosine: uptake mechanisms and clinical applications. Nucl Med Biol 2006;33:287-94.

37. Patel YC. Molecular pharmacology of somatostatin receptor subtypes. J Endocrinol Invest 1997;20:348-67.

38. Reichlin S. Somatostatin. N Engl J Med 1983;309:1495-501.

39. Cescato R, Schulz S, Waser B, Eltschinger V, Rivier JE, Wester H-J, Culler M, Ginj M, Liu Q, Schonbrunn A. Internalization of sst2, sst3, and sst5 receptors: effects of somatostatin agonists and antagonists. J Nucl Med 2006;47:502-11.

40. Ramírez C, Cheng S, Vargas G, Asa SL, Ezzat S, Gonzalez B, Cabrera L, Guinto G, Mercado M. Expression of Ki-67, PTTG1, FGFR4, and SSTR 2, 3, and 5 in nonfunctioning pituitary adenomas: a high throughput TMA, immunohistochemical study. J Clin Endocrinol Metab 2012;97:1745-51.

41. Frühwald MC, O'dorisio MS, Pietsch T, Reubi JC. High expression of somatostatin receptor subtype 2 (sst 2) in medulloblastoma: implications for diagnosis and therapy. Pediatr Res 1999;45:697.

42. Frühwald MC, Rickert CH, O’Dorisio MS, Madsen M, Warmuth-Metz M, Khanna G, Paulus W, Kühl J, Jürgens $H$, Schneider P. Somatostatin receptor subtype 2 is expressed by supratentorial primitive neuroectodermal tumors of childhood and can be targeted for somatostatin receptor imaging. Clin Cancer Res 2004;10:2997-3006.

43. Sharma P, Mukherjee A, Bal C, Malhotra A, Kumar R. Somatostatin Receptor-Based PET/CT of Intracranial Tumors: A Potential Area of Application for 68Ga-DOTA Peptides? AJR Am J Roentgenol 2013;201:1340-7.

44. Dutour A, Kumar U, Panetta R, Ouafik LH, Fina F, Sasi R, Patel YC. Expression of somatostatin receptor subtypes in human brain tumors. Int J Cancer 1998;76:620-7.

45. Reubi JC, Lang W, Maurer R, Koper JW, Lamberts SW. Distribution and biochemical characterization of somatostatin receptors in tumors of the human central nervous system. Cancer Res 1987;47:5758-64.

46. Reubi JC, Maurer R, Lamberts SW. Somatostatin binding sites in human leptomeninx. Neurosci Lett 1986;70:183-6.

47. Reubi JC, Maurer R, Klijn JG, Stefanko SZ, Foekens JA, Blaauw G, Blankenstein MA, Lamberts SW.

High incidence of somatostatin receptors in human meningiomas: biochemical characterization. J Clin Endocrinol Metab 1986;63:433-8.

48. Afshar-Oromieh A, Giesel FL, Linhart HG, Haberkorn U, Haufe S, Combs SE, Podlesek D, Eisenhut M, Kratochwil

C. Detection of cranial meningiomas: comparison of 68 Ga-DOTATOC PET/CT and contrast-enhanced MRI. Eur J Nucl Med Mol Imaging 2012;39:1409-15.

49. Milker-Zabel S, Zabel-du Bois A, Henze M, Huber P, Schulz-Ertner D, Hoess A, Haberkorn U, Debus J. Improved target volume definition for fractionated stereotactic radiotherapy in patients with intracranial meningiomas by correlation of CT, MRI, and [68Ga]DOTATOC-PET. Int J Radiat Oncol Biol Phys 2006;65:222-7.

50. Gehler B, Paulsen F, Öksüz MÖ, Hauser T-K, Eschmann SM, Bares R, Pfannenberg C, Bamberg M, Bartenstein P, Belka C. [68 Ga]-DOTATOC-PET/CT for meningioma IMRT treatment planning. Radiat Oncol 2009;4:56.

51. Packer RJ, Vezina G. Management of and prognosis with medulloblastoma: therapy at a crossroads.Arch Neurol 2008;65:1419-24.

52. Cervera P, Videau C, Viollet C, Petrucci C, Lacombe J, Winsky-Sommerer R, Csaba Z, Helboe L, Daumas- 
Duport C, Reubi J. Comparison of somatostatin receptor expression in human gliomas and medulloblastomas. J Neuroendocrinol 2002;14:458-71.

53. Malik D, Sood A, Mittal BR, Singh H, Basher RK, Shukla J, Bhattacharya A, Singh SK. Nonspecific uptake of 68Gaprostate-specific membrane antigen in diseases other than prostate malignancy on positron emission tomography/ computed tomography imaging: A pictorial assay and review of literature. Indian J Nucl Med 2018;33:317-25.

54. DeMarzo AM, Nelson WG, Isaacs WB, Epstein JI. Pathological and molecular aspects of prostate cancer. Lancet 2003;361:955-64.

55. Silver DA, Pellicer I, Fair WR, Heston W, CordonCardo C. Prostate-specific membrane antigen expression in normal and malignant human tissues. Clin Cancer Res 1997;3:81-5.

56. Chang SS, O'Keefe DS, Bacich DJ, Reuter VE, Heston WD, Gaudin PB. Prostate-specific membrane antigen is produced in tumor-associated neovasculature. Clin Cancer Res 1999;5:2674-81.

57. Sheikhbahaei S, Afshar-Oromieh A, Eiber M, Solnes LB, Javadi MS, Ross AE, Pienta KJ, Allaf ME, Haberkorn U, Pomper MG. Pearls and pitfalls in clinical interpretation of prostate-specific membrane antigen (PSMA)-targeted PET imaging. Eur J Nucl Med Mol Imaging2017;44:2117-36.

58. Bilgin R, Ergül N, Çermik TF. Incidental Meningioma Mimicking Metastasis of Prostate Adenocarcinoma in 68Ga-Labeled PSMA Ligand PET/CT. Clin Nucl Med 2016;41:956-8.

59. Afshar-Oromieh A, Malcher A, Eder M, Eisenhut M, Linhart H, Hadaschik B, Holland-Letz T, Giesel F, Kratochwil C, Haufe S. PET imaging with a [68 Ga] gallium-labelled PSMA ligand for the diagnosis of prostate cancer: biodistribution in humans and first evaluation of tumour lesions. Eur J Nucl Med Mol Imaging 2013;40:486-95.

60. Afshar-Oromieh A, Zechmann C, Eder M, Eisenhut M, Haberkorn U. [68Ga] Gallium labelled PSMA ligand as new PET-tracer for the diagnosis of prostate cancer: Normal uptake of healthy tissue. J Nucl Med 2012;53:1414-.

61. Eder M, Schäfer M, Bauder-Wüst U, Hull WE, Wängler C, Mier W, Haberkorn U, Eisenhut M. 68Ga-complex lipophilicity and the targeting property of a urea-based PSMA inhibitor for PET imaging. Bioconjug Chem 2012;23:688-97.

62. Fendler WP, Eiber M, Beheshti M, Bomanji J, Ceci F, Cho S, Giesel F, Haberkorn U, Hope TA, Kopka K. 68 Ga-
PSMA PET/CT: Joint EANM and SNMMI procedure guideline for prostate cancer imaging: version 1.0. Eur J Nucl Med Mol Imaging 2017;44:1014-24.

63. Kallur KG, Ramachandra PG, Rajkumar K, Swamy SS, Desai I, Rao RM, Patil SG, Sridhar P, Madhusudhan N, Krishnappa RS. Clinical utility of gallium-68 PSMA PET/CT scan for prostate cancer. Indian J Nucl Med 2017;32:110.

64. Jain TK, Jois AG, Singh S, Kumar R, Mittal B. Incidental detection of tracer avidity in meningioma in 68Ga-PSMA PET/CT during initial staging for prostate cancer. Rev Esp Med Nucl Imagen Mol 2017;36:133-4.

65. Kanthan GL, Izard MA, Emmett L, Hsiao E, Schembri GP. Schwannoma Showing Avid Uptake on 68Ga-PSMAHBED-CC PET/CT. Clin Nucl Med 2016;41:703-4.

66. Rischpler C, Maurer T, Schwaiger M, Eiber M. Intense PSMA-expression using ${ }^{\wedge}$ sup $68^{\wedge}$ Ga-PSMA PET/CT in a paravertebral schwannoma mimicking prostate cancer metastasis. Eur J Nucl Med Mol Imaging2016;43:193.

67. Vamadevan S, Le K, Shen L, Ha L, Mansberg R. Incidental Prostate-Specific Membrane Antigen Uptake in a Peripheral Nerve Sheath Tumor. Clin Nucl Med 2017;42:560-2.

68. Chan M, Hsiao E. Subacute Cortical Infarct Showing Uptake on 68Ga-PSMA PET/CT. Clin Nucl Med 2017;42:110-1.

69. Noto B, Vrachimis A, Schäfers M, Stegger L, Rahbar K. Subacute Stroke Mimicking Cerebral Metastasis in 68Ga-PSMA-HBED-CC PET/CT. Clin Nucl Med 2016;41:e449-51.

70. Wang W, Tavora F, Sharma R, Eisenberger M, Netto GJ. PSMA expression in Schwannoma: a potential clinical mimicker of metastatic prostate carcinoma. Urol Oncol 2009;27:525-8.

71. Krohn T, Verburg FA, Pufe T, Neuhuber W, Vogg A, Heinzel A, Mottaghy FM, Behrendt FF. [68 Ga] PSMAHBED uptake mimicking lymph node metastasis in coeliac ganglia: an important pitfall in clinical practice. Eur J Nucl Med Mol Imaging 2015;42:210-4.

72. Ghasemi M, Nabipour I, Omrani A, Alipour Z, Assadi M. Precision medicine and molecular imaging: new targeted approaches toward cancer therapeutic and diagnosis. Am J Nucl Med Mol Imaging 2016;6:310.

73. Collins FS, Varmus H. A new initiative on precision medicine. N Engl J Med 2015;372:793-5.

74. Hood L, Balling R, Auffray C. Revolutionizing medicine in the 21 st century through systems approaches. Biotechnol J 2012;7:992-1001. 
75. Hernandez R, Sun H, England CG, Valdovinos HF, Barnhart TE, Yang Y, Cai W. ImmunoPET imaging of CD146 expression in malignant brain tumors. Mol Pharm 2016;13:2563-70.

76. Wright BD, Lapi SE. Designing the magic bullet? The advancement of immuno-PET into clinical use. J Nucl Med 2013;54:1171-4.

77. Börjesson PK, Jauw YW, Boellaard R, de Bree R, Comans EF, Roos JC, Castelijns JA, Vosjan MJ, Kummer
JA, Leemans CR. Performance of immuno-positron emission tomography with zirconium-89-labeled chimeric monoclonal antibody U36 in the detection of lymph node metastases in head and neck cancer patients. Clin Cancer Res 2006;12:2133-40.

78. Reddy S, Robinson MK. Immuno-positron emission tomography in cancer models. Semin Nucl Med 2010;40:182-9.

Cite this article as: Shooli H, Dadgar H, Wáng YX, Vafaee MS, Rassaei Kashuk S, Nemati R, Jafari E, Nabipour I, Gholamrezanezhad A, Assadi M, Larvie M. An update on PETbased molecular imaging in neuro-oncology: challenges and implementation for a precision medicine approach in cancer care. Quant Imaging Med Surg 2019;9(9):1597-1610. doi: 10.21037/qims.2019.08.16 